\title{
Bottlenecks and Opportunities towards Achieving the Targeted 95-95-95 HIV Services in a Rural District in Eastern Uganda
}

Monkya Samuel Namenkere

Busitema University

Ayaa Mary Stella

Busitema University

Sukuku Linda

Busitema University

Kharono Juliet

Busitema University

Mugabi Charles

Busitema University

Chelangat Benina

Busitema University

Mary Abwola Olwedo

Soroti University

Carol Nabasumba

Busitema University

Paul Oboth

Busitema University

Julius Osele

Busitema University

Rebecca Nekaka

Busitema University

Jacob Stanley Iramiot ( $\sim$ jiramiot@gmail.com )

Busitema University

\section{Research Article}

Keywords: HIV, ART, viral load suppression, Uganda

Posted Date: November 4th, 2021

DOI: https://doi.org/10.21203/rs.3.rs-1019754/v1 
License: (c) (i) This work is licensed under a Creative Commons Attribution 4.0 International License. Read Full License 


\section{Bottlenecks and Opportunities towards Achieving the Targeted 95-95-95 HIV Services in a Rural District in Eastern Uganda.}

Monkya Samuel Namenkere ${ }^{1}$, Ayaa Mary Stella ${ }^{1}$, Sukuku Linda ${ }^{1}$, Kharono Juliet ${ }^{1}$, Mugabi Charles $^{1}$, Chelangat Benina $^{1}$, Mary Abwola Olwedo ${ }^{4}$, Carol Nabasumba ${ }^{1}$, Paul Oboth ${ }^{1}$, Julius Osele $^{3}$, Rebecca Nekaka ${ }^{1}$, and Jacob Stanley Iramiot ${ }^{2}$.

${ }^{1}$ Department of Community and Public health, Faculty of Health Sciences, Busitema University. P.O Box 1460, Mbale

${ }^{2}$ Department of Microbiology and Immunology, Faculty of Health Sciences, Busitema University. P.O Box 1460, Mbale

${ }^{3}$ Bukedea Health Centre IV

${ }^{4}$ Soroti University

\section{ABSTRACT}

Background: There are 1.3 million people living with HIV in Uganda of which $73 \%$ know their HIV-positive status, $67 \%$ of the HIV-positive patients on ART, and $60 \%$ are viral load suppressed. Uganda has made progress in reducing the HIV prevalence from $7.3 \%$ in 2011 to $6 \%$ in 2017, however, more needs to be done to meet the WHO target of $95 \%$ of the population knowing their HIV status, 95\% enrolled on treatment and 95\% achieving viral suppression. This study aimed to assess the bottlenecks and opportunities towards achieving the 959595 targeted HIV services in Bukedea district.

Methods: A mixed-methods cross-sectional study was conducted in Bukedea district covering males and females aged 18-65 years who had consented to participate in the study. We used a purposive sampling procedure to select our study participants. Qualitative data was collected through focus group discussions, key informant interviews, and document reviews for quantitative data. Quantitative data were analyzed using STATA v 14 whereas qualitative data were analyzed using the thematic analysis approach.

Results: The challenges were grouped as patient-related, medication-related, and facility-related challenges. The patient-related challenges were stigma, fear to take the drugs, poor nutrition, long distances, alcoholism, busy working schedules, domestic violence. The medication-related 
challenges were side effects, pill burden. The facility-related challenges were inadequate pretest counseling, stockouts. The use of anti-retroviral drugs was common in piggery and poultry and the sources of these drugs were reported to be the people on ART and the health workers.

The opportunities included home-based counseling, organizing more outreaches, counseling and health education, targeted testing, and strengthening the VHT networks.

Conclusion: The study revealed that the major challenges towards achieving the targeted 95-9595 HIV services were stigma, inadequate pre-test counseling, fear of disclosure, and poor adherence due to alcoholism, sharing of drugs with animals and partners. The use of Antiretroviral drugs in animal husbandry was common in Bukedea District. Continuous sensitization about HIV and the importance of adherence to drugs, continuous and adequate counseling of the clients on ART, and close monitoring of their viral load could help to improve enrollment into care, adherence to HIV treatment, and HIV viral load suppression.

\section{0: BACKGROUND}

A Global decline in the number of new HIV infections from 3.4 million in 2001 to 2.3 million, indicating a 33\% decline has brought new hope in the fight against HIV/AIDS (1) The reductions in new HIV infection has been greatly observed among newborn, decreasing from 530,000 to 260,000 in 2000 and 2013 respectively due to national and global efforts invested in

prevention of mother-to-child transmission (PMTCT) programs (1) Even with this progress, the number of HIV cases is steadily increasing with sub-Saharan Africa being the greatly affected region (2). Despite the high prevalence of HIV/AIDS in Africa, the Highly Active Anti-retroviral Therapy (HAART) coverage is still low due to some reasons (2). In Africa, an estimated 66\% of eligible people living with HIV were not receiving HAART in 2013 (3), only 45\% of adults living with HIV were reported to know their HIV status, $86 \%$ of diagnosed persons were initiated on HAART, and an estimated $76 \%$ of persons on ART had achieved HIV viral load suppression in 2013 (4). The Uganda Population-based HIV Impact Assessment (UPHIA) results revealed that the country has made significant progress in reducing the HIV prevalence from $7.3 \%$ in 2011 to $6 \%$ in 2017 . There are 1.3 million people living with HIV in Uganda of which $73 \%$ know their HIV-positive status,67\% of the HIV-positive patients on HAART, and $60 \%$ have achieved viral suppression (5). The World Health Organization set an ambitious 90-90-90 
target (6) which has now been upgraded to the 95, 95, 95 target; meaning 95\% of the people living with HIV should know their status, $95 \%$ of the diagnosed people enrolled into care, and 95\% of those on ART achieve viral load suppression. In response, the Uganda Ministry of Health set a 95, 95, 95 target. The progress of Uganda towards these targets is still worryingly low. Cases of low testing, poor adherence, and poor viral load suppression were common in Bukedea district. Our study assessed the bottlenecks and opportunities towards the targeted 95, 95, 95 HIV services in Bukedea district.

\section{MATERIALS AND METHODS}

\section{STUDY DESIGN}

A cross-sectional study was conducted in Bukedea district using mixed methods.

\section{STUDY AREA}

Bukedea district is found in Eastern Uganda, mainly inhabited by the Iteso tribe. It has a population of 203,600 people according to 2014 Census data, of which 98,684 (48.5\%) are males and 104,916 (51.5\%) are females, 74533 are aged 0-9 years, 53164 between 10-19 years,45151 between 20-39 years, 20367 between 40-59 and 10385 aged 60 and above years. The biggest population is people under 20 years of age, suggesting a youthful population. $96.7 \%$ of the population stays in rural areas and $3.3 \%$ urban places according to (7).

\section{SAMPLING PROCEDURE}

We reviewed existing information on HIV testing, enrolment to care and viral suppression from the HIV registers for Bukedea HCIV and also analyzed information on the Bukedea district dashboard to draw the general picture of the district's performance towards achieving the 95, 95, 95 WHO target.

Purposive sampling was used to select the key informant interview participants. The participants were selected based on the knowledge they had about the uptake of HIV services at Bukedea Health center IV and the district as a whole. Members of the village health team (VHTs), local leaders, and health care providers were selected to participate in this study. The sample size for the focus group discussions and the key informant interviews was determined by saturation 
method where the number was got after reaching a point where no new information was obtained.

\section{DATA COLLECTION PROCEDURE}

Data collection was through document review, Focus group discussions, and Key informant interviews. The focus group discussions were guided by the moderator who introduced topics for discussion to the participants who were given specific codes for identification. A total of 7 focus group discussions each consisting of 6-12 participants were conducted in the community.

Focus groups discussions involved people in the same age brackets and males and females were grouped separately. The Focus groups discussions were conducted with the VHTs and the community members to get data about the bottlenecks and the opportunities towards achieving the 95, 95, 95 HIV service at Bukedea district.

The Key informant interviews were also used in collecting data from the health care workers and HIV clients. Key informants were carefully selected based on the knowledge they had about the uptake of HIV services in the community. The key informants included the health workers of the health facility for example the ART in charge, the counselor, linkage facilitator, HIV viral load suppressed and non-suppressed clients. To assess the prevalence of HIV viral load nonsuppression, we reviewed the ART register and the HIV viral load non suppressed clients' register and analyzed the Bukedea district dashboard.

\section{Inclusion criteria.}

Males and females aged 15-65 years were selected for the study. For HIV testing, both HIV positive and negative people were selected. For HIV treatment and viral load suppression, only HIV positive people currently on ART were selected

\section{Data Quality Control}

This was part and parcel of the research process right from data collection to data analysis. During the focus group discussion, the moderators ensured balanced chances of participation amongst all the participants. The separation of the focus group participants minimized the influence of the power dynamics on the information collected. 
The tools used for the focus group discussions and key informant interviews were pre-tested before the actual data collection to ensure data quality. Member checking was also done to ensure that accurate information was collected.

\section{Data analysis}

Thematic data analysis was used. After data collection, the data was transcribed that is, interpreted, and translated into words that could be studied and coded. The transcribed data was then coded. After coding, all the codes and data extracts were collated for the later stages of data analysis. Searching for themes was then done from the coded data that involved examining the codes and collated data to identify significant broader patterns of meaning. The themes were then reviewed by checking the candidate themes to ensure that a convincing story of the data was got and to ensure that the data was in line with the research objectives. The themes were then named and defined by a detailed analysis of each theme, working out the scope and focus of each theme, determining the story of each theme. The final phase of data analysis involved writing the final report. The researchers then present the findings and interpretations of the data

\section{Ethical Consideration}

Ethical clearance was sought from the Busitema University Faculty of Health Sciences Institutional Research Board. Confidentiality of the respondents was ensured and their names appeared nowhere instead numbers were used for identification during the focus group discussions. Informed consent was also obtained from the respondents and this was evidenced by a signature or thumbprint on the key informant guide.

\section{RESULTS}

\section{Study participants}

Ninety participants took part in the study. Of the 90 participants, 48 were community members, 12 were VHTs, 3 were health care providers that included the ART in charge, linkage facilitator, and the counselor, 12 participants were Viral load non suppressed patients and 15 were viral load suppressed patients. 7 Focus group discussion sessions and 30 face-to-face key informant interviews were conducted. 
Twenty-Four community members that participated in the study were females and 24 were males, 32 of the community members were aged 20-65 years, 16 of them were aged between 18 and 20 years. The clients that participated in the study were those who had been enrolled in HIV care, 12 of them were HIV viral load suppressed clients and 15 were HIV viral load nonsuppressed clients. Three health care providers; the ART in charge, counselor, and linkage facilitator were interviewed Twelve VHTs participated in the Focus group discussions.

\section{HIV TESTING}

The bottlenecks that emerged from the focus group discussions with community members and VHTs were classified as the client-related and facility-related factors. The client-related bottlenecks included; long distances to the health facility offering HIV care services, the fear of disclosure of the results to partners, stigma, and the busy working schedules. The facility-related bottlenecks cited by the participants were bad experiences from the facility.

Stigma was commonly cited in the focus group discussions with the adolescents in the community. The stigma was mainly due to fear for their colleagues at school to know about them being positive and also fear of being started on drugs

"HIV is a greatly feared disease in our community. People do not want to test for it and those who are tested and found positive refuse to be started on medication. I think the major cause of that is fear for their colleagues knowing about it and also fear to take the drugs.',

The community members frequently mentioned that the fear of disclosure of the positive HIV results to their marriage partners made them not test for HIV. This was common among the females who feared intimidation from their husbands in case they were found positive.

"Here in Kachabule, a husband badly beat up his wife because she tested positive without informing him, on informing the husband about the results, he just blamed the lady, beat her up, and chased her away. This makes the wives fear to test".

Some community members confessed that the busy working schedules they had, made them not turn up for testing because they work from morning up to evening as illustrated below. 
"I'm a carpenter and I do my work from morning to evening to get money for survival at home. I hardly get the time to go to the health center for testing and yet the health center is even far. Any time I miss not working is money being missed".

Some people in the community did not test because of the long distances to the health facility. When asked about community outreach programs, they said that they are not aware of any outreach programs that take place in their villages.

Table 1: Health Service Coverage in Bukedea District

\begin{tabular}{|l|l|l|l|l|}
\hline HF Level & \multicolumn{2}{|l|}{ Facility ownership } & \multicolumn{2}{l|}{ Accreditation for ART services } \\
\hline & Govt. & PNFP & Govt. & PNFP \\
\hline Hospitals & 00 & 00 & 00 & 00 \\
\hline HC IV & 01 & 00 & 01 & 00 \\
\hline HC III & 05 & 00 & 05 & 00 \\
\hline HC II & 01 & 05 & 00 & 02 \\
\hline Total & $\mathbf{0 7}$ & $\mathbf{0 5}$ & $\mathbf{0 6}$ & $\mathbf{0 2}$ \\
\hline
\end{tabular}

FH=Health Facility, Govt=Government, PNFP=Private Not For Profit.

The Uganda health system is organized in a hierarchical manner according to the population they serve. The National Referral Hospital serves a population of 30,000,000, the Regional Referral Hospital-2,000,000, a General hospital-500,000, the Health Center IV -70,000, the Health Center III 20,000, the Health Center II-5,000 and the Health Center I (Village Health Team)-1,000 people (8).

The focus group discussions cited deterrent costs which they incur when the health facilities lack gloves and other sundries. All focus group discussions cited this challenge.

"There is a time I went to the health center when I was suffering from malaria, they told me to buy gloves because since they had got over. From that time, I have never gone back to the facility and also not yet tested."

\section{ENROLLMENT INTO HIV CARE}

The bottlenecks in enrolment to care (the second 95) were also patient-related and facilityrelated. The patient-related challenges were fear of disclosure to the partners, fear to take the 
drugs, failure to believe in the positive results. The health care providers frequently mentioned that some people who turn positive after testing refuse to get enrolled into care because of fear to disclose the information to their partners. Even the ones that are started on treatment often hide the drugs from their partners. Based on the fact that HIV treatment is for life, some people who test positive feared to be started on drugs. The ART In-charge mentioned that due to the fear, some patients often said that they need time to think about whether to begin taking the medication or not. Some people take time to believe the positive turned HIV results and end up running away from the facility.

"A patient came, he was tested positive, the patient did not accept that the results were his. He decided to move around various facilities testing to confirm if the results were positive. But after, he came back after some time because he had some complications and had to be started on ART."

The only facility-related challenge was inadequate pre-test counseling. This was reported by the counselor. The ART in charge said that inadequate pre-test counseling was because there were many testing points and only one counselor was attached to the facility.

\section{HIV VIRAL LOAD SUPPRESSION.}

The Bukedea HCIV had nine hundred sixty clients enrolled on ART at the time of this study.

Table 2: Viral load suppression at Bukedea HCIV

\begin{tabular}{|l|l|l|l|}
\hline Demographics & Number & Suppressed (\%) & Non- suppressed (\%) \\
\hline Sex & & & \\
\hline Male & $381(39.7)$ & $323(43.7)$ & $58(56.3)$ \\
\hline Female & $579(60.3)$ & $534(56.3)$ & $45(43.7)$ \\
\hline Age (years) & & & \\
\hline$<15$ & $252(26)$ & $234(93)$ & $18(7)$ \\
\hline $15-25$ & $258(27)$ & $248(96)$ & $10(4)$ \\
\hline $26-65$ & $401(42)$ & $330(82)$ & $71(18)$ \\
\hline Above 65 & $49(5)$ & $45(92)$ & $4(8)$ \\
\hline
\end{tabular}


There was a higher number of females attending the HIV clinic than the males (Table 2). The HIV viral non suppression was higher among the adult population (26/65 years) and lower among the children and young adults below 25 years.

The bottlenecks in HIV suppression were classified as patient-related, facility-related challenges, and medication-related challenges. The patient-related bottlenecks included stigma and discrimination, alcoholism, poor nutrition, sharing of drugs with animals and partners, genderbased violence, lack of family support, religious beliefs, multiple partners, and forgetfulness about taking the drugs.

Patients and the health care workers frequently reported that stigma and discrimination made the patients stop taking the pills. Such patients ended up abandoning the drugs as illustrated in the quote below.

"I used to miss my pills because of the people I used to stay with. My boyfriend did not know about my status and I was worried about revealing the truth because I did not want us to break up. I worry about how they may perceive it if they get to know that I'm on pills."

Some women with undisclosed HIV status would hide their pills in odd areas such as inside the grass in the grass thatched house where drugs sometimes get wet in the rain season for the fear of their husbands discovering that they are taking pills. Some of the female participants confessed keeping their pills under the cooking stones where their husbands are least expected to check and find them.

Alcoholism also came out strongly as a driver of non-adherence to the medication and hence non-suppression. Alcohol consumption was common among both men and women and in many occasions, children.

"I'm an askari, sometimes I take beer with my colleagues the moment I receive my salary just to be happy with them"

From the Focus group discussions in the community, there were several mentions of people living with sharing drugs with animals. The ARVs were given to pigs as fatteners and to chicken as a remedy for avian viral infections. Sharing ARVs was also reported to be common among Spouses. Some reasons given for the sharing of ARVs were; the busy schedule of the men, long 
lines in the health facilities and that men were afraid of being seen picking their pills. This was frequently mentioned in the focus group discussions in the community as quoted below;

"Here in Kachabule, there was a man who used to give ARVs to the pigs to make them grow fat and to hens to treat coccidiosis and I know many couples that share drugs especially those on the same regimen."

One of the key informants (the counselor) mentioned that gender-based violence greatly contributed to non-adherence. The commonly affected were the females who were tortured by their husbands which caused stress.

Some wives abandon the drugs because of family conflicts with their husbands. Some who divorce leave the drugs behind. This makes them abandon their medication.

Some patients confessed that they missed medications simply because of forgetfulness about the next day of picking the refills and also the time at which to take the drugs. The natures of work and alcohol consumption especially among the security personnel, also often referred to as "askaris"'were reported to be the common cause of skipping medication and refill appointments.

"With the nature of my work, I have to wake up very early in the morning and report to duty because I'm an askari, sometimes I'm deployed to work in the nights and this makes me miss some days especially when my phone is off to check for the time."

Patients said that one of the limiting factors to taking the drugs was lack of food to eat.

"We have been in the dry season, there are days we could only afford one meal a day, and this makes it hard for me to take the drugs because I could not take them with nothing to eat yet the drugs are too strong."

Some of the people living with HIV (PLWH) reported that they did not have support from the family members and this greatly contributed to the skipping of medication.

" When I'm with my wife, I sometimes forget to take the medication and I eat poorly but when I'm taken to my sister's place I find no problem in taking the drugs and I feel better.' 
The community members and the adherence counselor reported that some patients who were on ART abandoned it just because their religious leaders told them that even with no medication, Jesus would heal them.

The other medication-related challenges included the pill burden, side effects, resistance and the pill burden.

"I stopped taking the drugs because I was tired of waking up every early morning which was so inconveniencing."

Adverse effects for example nightmares, confusion, dizziness, vomiting, and rashes on the skin made some patients stop taking the medication as illustrated below from an interview with a nonsuppressed client.

"From 2013, I was not having any challenges till recently when I began getting side effects for example dizziness. The challenge that I think is bringing about this is the shortage of food. I sometimes have nothing to eat and when I swallow the drugs, I begin feeling dizzy'”

One of the Key informants reported that there were a few cases of the regimen failure for the patients especially those on second-line treatment. Stock-outs of vacutainers, loss to follow-ups were also common occurrences at the health facility.

"There was a time they had stockouts for vacutainers and this made viral load monitoring for the patients and CD4 counts hard at the facility. The option they had was to refer the patients to other health facilities to check for their viral loads. " 


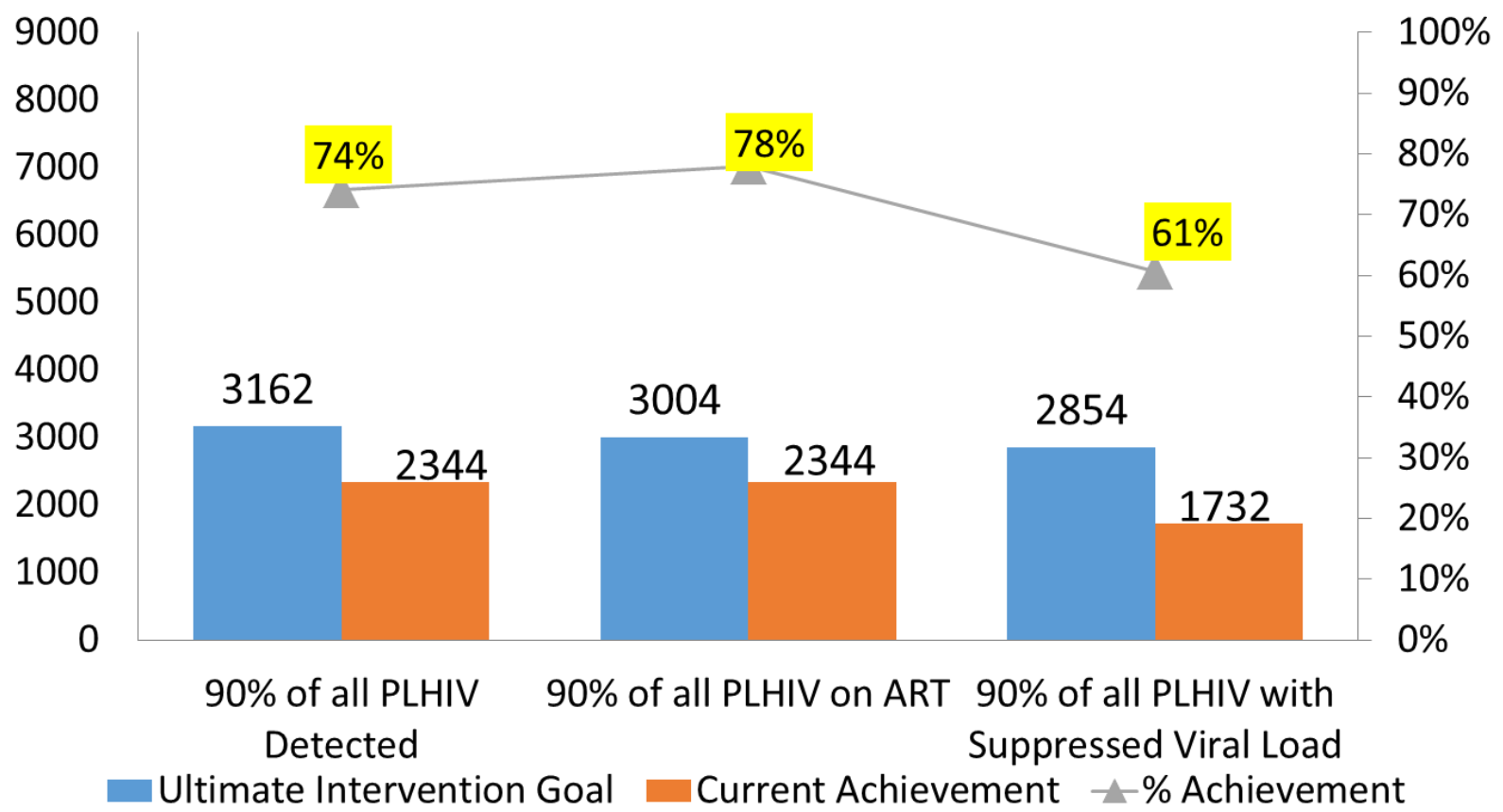

Fig. 1: The Progress of Bukedea district towards the 90-90-90 Global Targets.

This graph highlights the previous WHO targets of 90-90-90. The performance of Bukedea district was still below the WHO set previous targets (Fig.1). Whereas the district HIV detection and enrolment rates were getting closer to the previous targets, the viral suppression rates were still much lower.

\section{OPPORTUNITIES}

From the focus group discussions and Key informative interviews, we had with the community members, patients, VHTs, and health workers, we were able to identify several opportunities that could be utilized to achieve the targeted 95-95-95- HIV services in Bukedea district

Counseling was seen as one of the ways stigma would be reduced and one of the factors that would contribute to adherence to treatment. At the facility, pre-test counseling was not adequately done and this explains why some people feared to be started on medication. Health education about the importance of taking the medication, the ways of ensuring proper adherence to treatment. Sensitization on the dangers of alcohol consumption could be used as an opportunity to improve adherence to treatment and keeping the appointments for drug refills.

Targeted testing was also identified as one of the ways of ensuring that all the people living with HIV get tested. The targeted testing was done through testing the people more at risk of 
contracting the virus (HIV) for example the commercial sex workers, the truck drivers, the private security personnel, police officers, prisoners, and substance abusers.

Although Assisted Partner Notification (APN) was being done, it was, unfortunately, being limited by inadequate funding. Assisted Partner notification ensures that all the sexual partners to a positive client are screened and tested for HIV.

Family tracking was seen as one of the ways of making sure all people living with HIV test and get to know their status (Fig. 2). This involves knowing the HIV statuses of the family members and young ones where the positive client comes from because of the possibility that they could have been born with the virus.

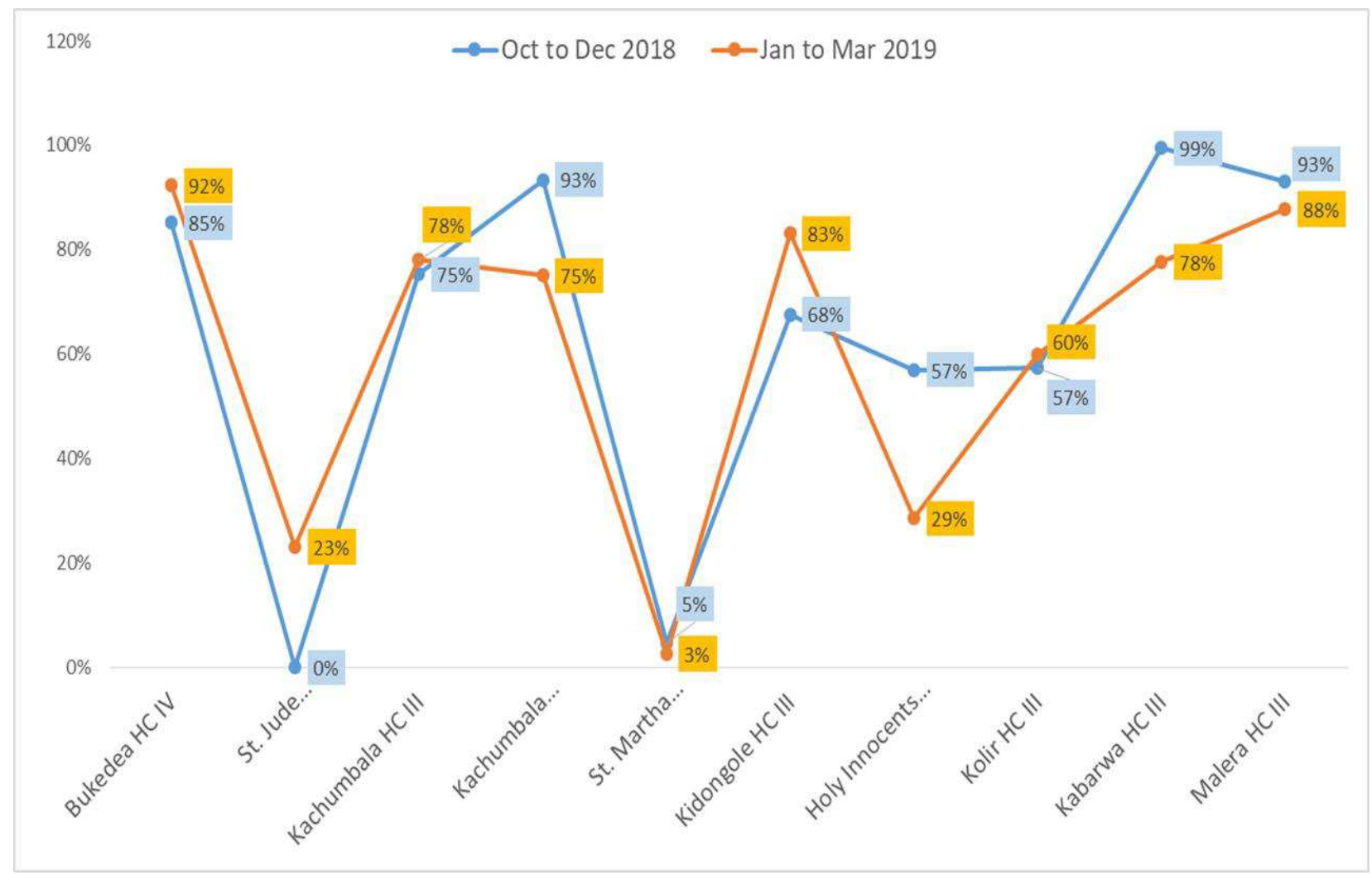

\section{Fig.2: Male Partner Testing in Bukedea District}

From our study, we found out that self-disclosure by the expert viral load suppressed HIV clients on ART would help reduce the fear for stigma and also make the PLWH to believe that once all the instructions for taking the medication are followed, a person living with HIV can have better living and get viral load suppression. 
Many people in the community said that they had not tested because of fear to be seen at the health facility while testing. We saw home to home testing as one of the opportunities to make sure that all the people living with HIV get to know their status.

Many people were not adhering to treatment because of gender-based violence and lack of family support. Linkage to social support networks ensures that the affected people are supported emotionally, financially, or in any other form of support.

VHTs played a major role in providing counseling, ensuring that the clients in their communities keep appointments and go for refills, and also picked refills for those who were unable to pick them because of fear and any other challenges.

One of the reasons patients gave for missing medications was because of forgetfulness about taking the medication. Setting alarms on phones for the clients who have them was seen as one of the ways to ensure that patients do not forget about taking the medications.

One of the reasons why some people feared to be enrolled into care and also abandoned the drugs was fear to disclose the status to the partners. We saw couple testing (Fig.3) as one of the ways of reducing the challenge because, through adequate pretest counseling, the married partners get ready for either accordant or discordant results.

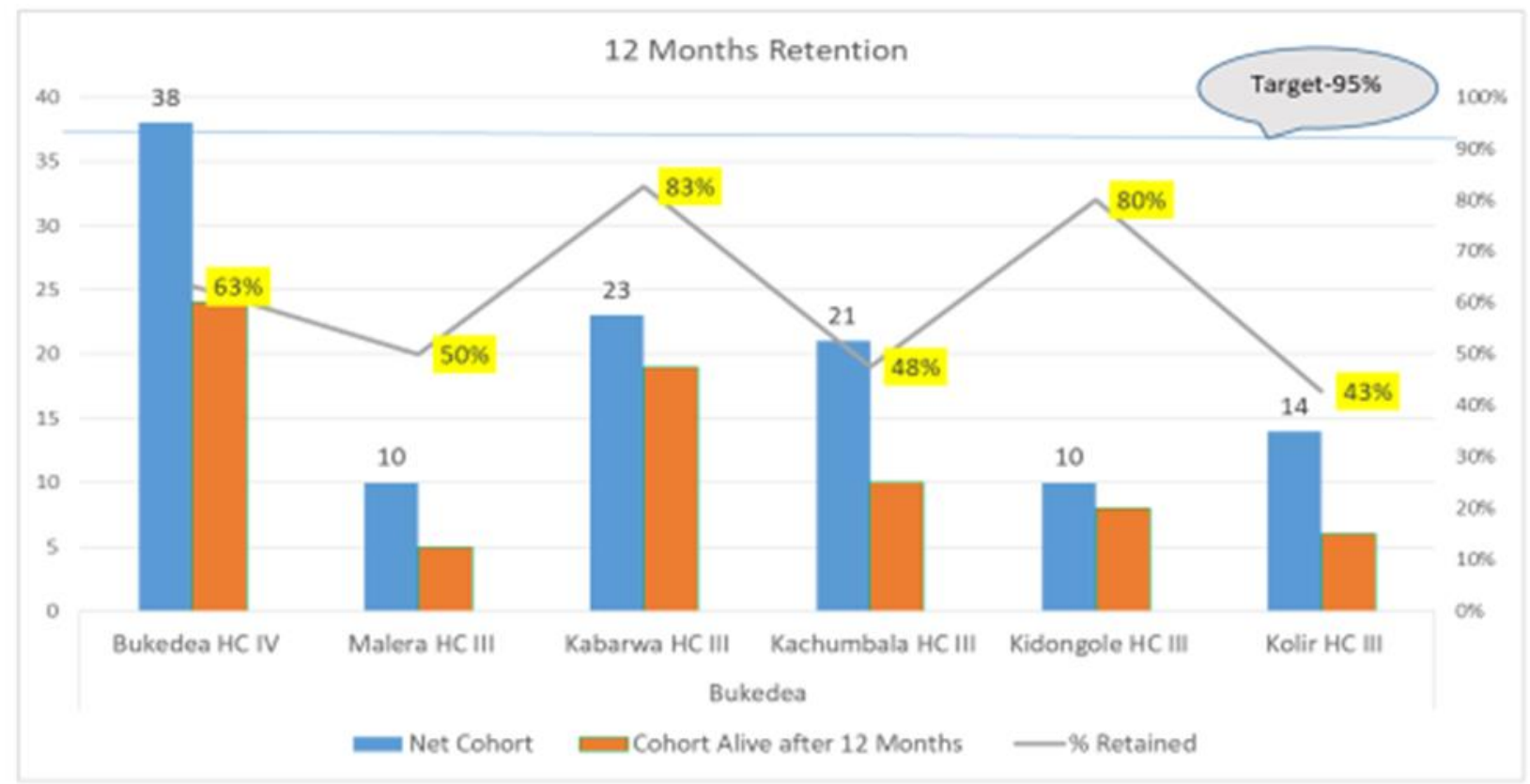

Fig.3: The 12 months' Retention to care 


\section{DISCUSSION}

Our study sought to identify the challenges and opportunities towards achieving the targeted 9595-95 HIV services in Bukedea district. The challenges were classified as client-related, facilityrelated, and medication-related challenges. The challenges people face in getting tested for HIV included stigma, fear of disclosure of results to partners, long distances, and busy working schedules.

Stigma, fear of disclosure of results, and long distances were identified as the major challenges that people encounter in getting to know their HIV status. Stigma was majorly reported from adolescent focus group discussions which were due to fear of their friends knowing about their positive HIV status and fear of the lifelong drugs. Fear of disclosure of results was majorly reported by married females who feared intimidation from their husbands and also losing their marriages in case they were found positive. The study also found out that long distances to the facility contributed to the poor turn up for the HIV testing services as reported by community members in the focus group discussions. These findings are similar to the results from a study carried out in 19 Ugandan districts to assess the availability, access, and utilization of HIV services (9). Results from several studies also reveal stigma, long distances, and fear of disclosure of results to partners being the major challenges faced by people in getting tested for HIV.

An important new finding this study found out was busy work schedules are a major hindrance to HIV testing as many people reported that they were always busy throughout the day and hardly get time to go and take HIV tests.

For enrollment into care, the challenges found out were fear to take the drugs, fear of disclosure of the results, failure to accept the positive results, and inadequate pretest counseling. Basing on the fact that ARVs are taken for a lifetime, many people who tested positive for HIV were not enrolled in care for fear of the bill burden. This is also due to fear of social discrimination as revealed by a study carried out in Bangladesh, Indonesia, and Vietnam to assess facilitators and barriers to retention in HIV care (10). Most married females refused to be enrolled into care for fear that they will be beaten up by their husbands in case the results came out positive and go back home with ARVs. 
An important new finding from this study is inadequate pretest counseling at the health facility. Adequate pretest counseling prepares one's mind to receive the results and the clients enroll into care with adequate information. The challenge was majorly reported by the community members in the focus group discussions. The ART in charge attributed this to the fact that the health facility has many testing points with only one counselor attached to the facility making adequate pretest counseling a challenge.

For HIV viral load suppression, the challenges were alcoholism, stigma, and discrimination, sharing drugs with animals and partners, gender-based violence, pill burden, side effects, and resistance. The study found out that, alcoholism greatly contributes to poor HIV viral load suppression as confessed by some of the clients during the key informant interviews. After taking alcohol, these clients get drunk and forget to take their drugs hence affecting their treatment adherence. Alcoholism also changes the eating habits of HIV clients a factor that greatly contributes to poor adherence. Some clients attributed poor adherence to pill burden as they have many ARV tablets and for life. A similar study in Zimbabwe about the failure of viral load suppression among adolescents on ART in Zimbabwe found out that the factors that contributed to the failure of viral load suppression among the clients included alcoholism, poor adherence, smoking, non-disclosure of the HIV status, and having more than one sexual partner for the previous twelve months (11).

Stigma and discrimination remain a problem that was greatly reported by most of the clients who even confessed to abandoning their medication because of the community's perception of them. Due to fear of stigma and social discrimination, the clients end up abandoning the treatment leaving them to non-adherence. A similar study conducted in rural Uganda and Kenya found out that the challenges to achieving viral load suppression among children and adults in rural Uganda and Kenya included stigma and structural barriers to care for example long wait times, frequent visits, opportunity costs to work, and inconvenience all of which degrade retention in care hence poor adherence to treatment thus resulting in low viral load suppression (12).

Religious beliefs also majorly contributed to non-adherence to treatment. This was common amongst the clients who receive healing prayers from their religious leaders that make them ignore the drugs leading to viral load non-suppression. A similar study conducted in Uganda to assess HIV viral load failure among perfect adherent HIV-positive adolescents showed that the 
rates of viral load suppression were higher among the adolescents who were adherent. However, $71 \%$ of the adherent adolescents had failed viral load suppression. The challenge to achieving viral load suppression among the adolescents was low adherence for people with strong spiritual beliefs who tend to miss medication during fasting and after receiving healing prayers and long exposure to Antiretroviral therapy (13).

An important new finding in this study is sharing drugs with animals and partners which was reported by some participants during the focus group discussions and key informant interviews. Some of the reasons they gave for giving the drugs to animals were to enhance fattening in pigs and prevent diseases in chickens. The clients who confessed sharing drugs with partners reported that the long distance to the facility made them share drugs so that they could both come for their refills at the same time.

Another important finding from this study is gender-based violence which was reported by the HIV counselor at the facility and study participants in the focus group discussions as one of the causes of poor adherence to treatment and majorly affected female clients who undergo psychological torture and stress. This makes them abandon the medication making hence poor adherence and viral load suppression.

The opportunities towards achieving the targeted HIV services included, home-based testing, targeted testing family tracking, counseling and health education, assisted partner notification, setting reminders, couple testing, linkage to social support networks, self-disclosure, strengthening the VHT network, and demonstrations on alcohol effects.

Routine testing and counseling by the health care providers increases awareness about the importance of HIV testing and also encourages the clients to adhere to the medication because they get to know the importance of taking the medicine. This finding is similar to one from a study conducted in Uganda, Tanzania, and South Africa to assess factors strengthening HIV testing access (14).

Another important opportunity is strengthening the VHT network through training them to help disseminate information to the community members through meetings and home visits hence increasing awareness about HIV. This is in line with a study conducted in rural Uganda to assess 
the perceived HIV testing norms. This study also suggests strengthening increased awareness about HIV testing through radio messages, billboards, and short text messages (15).

Self-disclosure of the HIV status by the expert clients especially during intensive adherence counseling sessions and health education talks to the HIV clients leads to a reduction of fear to take the drugs and also fear for social discrimination amongst the HIV clients. Self-disclosure is consistent with findings from a study conducted in Harare city Zimbabwe (11). The same study also revealed that social support networks give support to the clients for example affected by gender-based violence, food shortages, unemployed, and those with no family support (11).

Home-based testing was also frequently mentioned in focus group discussions as one of the opportunities to increase uptake services. This helps to address the challenge of fear of confidentiality and also long distances to the health facility. A similar study conducted in southwestern Uganda about expanding HIV linkage into care found out that training of community extension workers led to home-based HIV testing (16).

New important opportunities from our study include couple testing, assisted partner notification, and demonstrations about alcohol effects. Demonstration about the alcohol effects of the clients addicted to alcohol helped reduce the alcoholism challenge. This was done by putting one set of ARVs in water and the other set in alcohol. The one in water immediately dissolves and that in alcohol doesn't dissolve. This helped reduce alcoholism and increased proper adherence to medication. Couple testing ensures with adequate couple pretest counseling reduces the challenge of fear of disclosure of the results to the marriage partners and also prepares the couple for the HIV results as a couple. The other important opportunity from this study is Assisted Partner notification. This involves following up all the sexual partners of a person who tested positive and ensuring that all of them are screened and tested for HIV.

\section{CONCLUSION AND RECOMMENDATIONS}

The study revealed that the major challenges towards achieving the targeted 95-95-95 HIV services were stigma, inadequate pre-test counseling, fear of disclosure, and poor adherence due to alcoholism, sharing of drugs with animals and partners. Therefore, continuous sensitization about HIV and the importance of adherence to drugs, continuous and adequate counseling of the 
clients on ART, and close monitoring of their viral load could help to improve enrollment into care, adherence to HIV treatment, and HIV viral load suppression.

\section{Acknowledgements}

We are grateful to our research participants who volunteered information to us and the In-charge Bukedea HCIV for the support in the data collection process.

\section{Author contributions}

MSN, AMS, SL, KJ, MC and CB concieved the idea, participated in data collection and wrote the first draft of the manusript, CN, PO, JO, RN, and JS did data curation, supervised data collection and conducted critical reviews. All authors read and approved the final version to be published.

\section{Funding}

Research reported in this publication was supported by the Fogarty International Center of the National Institutes of Health, U.S. Department of State's Office of the U.S. Global AIDS Coordinator and Health Diplomacy (S/GAC), and President's Emergency Plan for AIDS Relief (PEPFAR) under Award Number 1R25TW011213. The content is solely the responsibility of the authors and does not necessarily represent the official views of the National Institutes of Health."

\section{Availability of data and materials}

All data on which conclusions of this manuscript are drawn has been included in this manuscript and whatever may be missing is available on request from the corresponding author.

\section{Competing interests}

The authors have declared that there are no competing interests.

\section{Consent for publication}

Not applicable 


\section{REFERENCES}

1. Diese M, Shrestha L, Pradhan B, Singh D, Raaijmakers H, Kisesa A, et al. Bottlenecks and opportunities for delivering integrated pediatric HIV services in Nepal. Curr Opin HIV AIDS. 2016;11 Suppl 1(Suppl 1):S21-S9.

2. Tessema B, Biadglegne F, Mulu A, Getachew A, Emmrich F, Sack U. Magnitude and determinants of nonadherence and nonreadiness to highly active antiretroviral therapy among people living with HIV/AIDS in Northwest Ethiopia: a cross - sectional study. AIDS Research and Therapy. 2010;7(1):2.

3. Alyssa Bilinski, Ermyas Birru, Matthew Peckarsky, Michael Herce, Noel Kalanga, Christian Neumann, et al. Distance to care, enrollment and loss to follow-up of HIV patients during decentralization of antiretroviral therapy in Neno District, Malawi: A retrospective cohort study PLOS 2017.

4. Novitsky V, Gaolathe T, Mmalane M, Moyo S, Chakalisa U, Yankinda EK, et al. Lack of Virological Suppression Among Young HIV-Positive Adults in Botswana. Journal of acquired immune deficiency syndromes (1999). 2018;78(5):557-65.

5. Uganda Go. Uganda HIV/AIDS Country Progress Report July 2016-June 2017; Theme:"Reaching men, girls and young women to reduce new HIV infections. UNAIDS - Joint United Nations Programme on HIV/AIDS; 2018.

6. Sidibé M, Loures L, Samb B. The UNAIDS 90-90-90 target: a clear choice for ending AIDS and for sustainable health and development. Journal of the International AIDS Society. 2016;19(1):21133.

7. Statistics UBo. The National Population and Housing Census 2014.

8. Ahebwa Amelia, Akol Walter, Achong Emmanuel, Mugerwa Timothy, Nakayenga Aminah, Omega Phillip, et al. Awareness of Antimicrobial Resistance among Primary Health Care Workers in Buyende District, Rural Eastern Uganda. Microbiology Research Journal International. 2017;22(5):1-11.

9. Bajunirwe F, Tumwebaze F, Akakimpa D, Kityo C, Mugyenyi P, Abongomera G. Towards 90-90-90 Target: Factors Influencing Availability, Access, and Utilization of HIV Services-A Qualitative Study in 19 Ugandan Districts. BioMed Research International. 2018;2018:9619684.

10. Koirala S, Deuba K, Nampaisan O, Marrone G, Ekström AM. Facilitators and barriers for retention in HIV care between testing and treatment in Asia-A study in Bangladesh, Indonesia, Lao, Nepal, Pakistan, Philippines and Vietnam. PloS one. 2017;12(5):e0176914.

11. Sithole Z, Mbizvo E, Chonzi P, Mungati M, Juru TP, Shambira G, et al. Virological failure among adolescents on ART, Harare City, 2017- a case-control study. BMC Infectious Diseases. 2018;18(1):469.

12. Kwarisiima D, Kamya MR, Owaraganise A, Mwangwa F, Byonanebye DM, Ayieko J, et al. High rates of viral suppression in adults and children with high CD4+ counts using a streamlined ART delivery model in the SEARCH trial in rural Uganda and Kenya. Journal of the International AIDS Society. 2017;20(Suppl 4):21673-.

13. Natukunda J, Kirabira P, Ong KIC, Shibanuma A, Jimba M. Virologic failure in HIVpositive adolescents with perfect adherence in Uganda: a cross-sectional study. Tropical Medicine and Health. 2019;47(1):8.

14. McNaghten AD, Schilsky Mneimneh A, Farirai T, Wamai N, Ntiro M, Sabatier J, et al. Implementation and Operational Research: Strengthening HIV Test Access and Treatment 
Uptake Study (Project STATUS): A Randomized Trial of HIV Testing and Counseling Interventions. Journal of acquired immune deficiency syndromes (1999). 2015;70(4):e140-e6.

15. Perkins JM, Nyakato VN, Kakuhikire B, Mbabazi PK, Perkins HW, Tsai AC, ..., et al. Actual versus perceived HIV testing norms, and personal HIV testing uptake: a cross-sectional, population-based study in rural Uganda. AIDS and Behavior. 2018;22(2):616-28.

16. Asiimwe S, Ross JM, Arinaitwe A, Tumusiime O, Turyamureeba B, Roberts DA, et al. Expanding HIV testing and linkage to care in southwestern Uganda with community health extension workers. Journal of the International AIDS Society. 2017;20(Suppl 4):21633. 